\section{(2) OPEN ACCESS}

\title{
Helicobacter pylori infection has a detrimental impact on the efficacy of cancer immunotherapies
}

\author{
Paul Oster, ${ }^{1}$ Laurie Vaillant, ${ }^{1}$ Erika Riva, ${ }^{1}$ Brynn McMillan, ${ }^{1}$ Christina Begka, ${ }^{1}$ \\ Caroline Truntzer, ${ }^{2}$ Corentin Richard, ${ }^{3}$ Marine M Leblond ${ }^{4}$ Meriem Messaoudene, ${ }^{3}$ \\ Elisavet Machremi, ${ }^{1}$ Emeric Limagne, ${ }^{2}$ Francois Ghiringhelli $\odot{ }^{2}{ }^{2}$ Bertrand Routy, ${ }^{3}$ \\ Gregory Verdeil, ${ }^{4}$ Dominique Velin (1) ${ }^{1}$
}

- Additional supplemental material is published online only. To view, please visit the journal online (http://dx.doi.org/ 10.1136/gutjnl-2020-323392).

'Service of Gastroenterology and Hepatology, Centre Hospitalier Universitaire Vaudois, University of Lausanne, Lausanne, Switzerland ${ }^{2}$ Department of Medical Oncology, Centre Georges François Leclerc, Dijon, France ${ }^{3}$ Research Centre for the University of Montréal (CRCHUM), HematologyOncology Division, Department of Medicine, University of Montreal Healthcare Centre (CHUM), Montreal, Quebec, Canada

${ }^{4}$ Department of Oncology, Centre Hospitalier Universitaire Vaudois, University of Lausanne, Lausanne, Switzerland

\section{Correspondence to} Dr Dominique Velin, Service of Gastroenterology and Hepatology, Centre Hospitalier Universitaire Vaudois, University of Lausanne, 1011 Lausanne, Switzerland;

dominique.velin@chuv.ch

LV and ER contributed equally.

Received 17 October 2020 Accepted 24 June 2021

\section{Check for updates}

(C) Author(s) (or their employer(s)) 2021. Re-use permitted under CC BY-NC. No commercial re-use. See rights and permissions. Published by BMJ.

To cite: Oster P, Vaillant $L$,

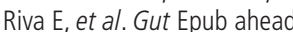
of print: [please include Day Month Year]. doi:10.1136/ gutjn-2020-323392

\section{ABSTRACT \\ Objective In this study, we determined whether Helicobacter pylori (H. pylori) infection dampens the efficacy of cancer immunotherapies.}

Design Using mouse models, we evaluated whether immune checkpoint inhibitors or vaccine-based immunotherapies are effective in reducing tumour volumes of $H$. pylori-infected mice. In humans, we evaluated the correlation between $H$. pylori seropositivity and the efficacy of the programmed cell death protein 1 (PD-1) blockade therapy in patients with non-small-cell lung cancer (NSCLC).

Results In mice engrafted with MC38 colon adenocarcinoma or B16-OVA melanoma cells, the tumour volumes of non-infected mice undergoing anticytotoxic T-lymphocyte-associated protein 4 and/ or programmed death ligand 1 or anti-cancer vaccine treatments were significantly smaller than those of infected mice. We observed a decreased number and activation status of tumour-specific $C D 8^{+} T$ cells in the tumours of infected mice treated with cancer immunotherapies independent of the gut microbiome composition. Additionally, by performing an in vitro co-culture assay, we observed that dendritic cells of infected mice promote lower tumour-specific $C D 8^{+} \mathrm{T}$ cell proliferation. We performed retrospective human clinical studies in two independent cohorts. In the Dijon cohort, H. pylori seropositivity was found to be associated with a decreased NSCLC patient survival on anti-PD-1 therapy. The survival median for $H$. pylori seropositive patients was 6.7 months compared with 15.4 months for seronegative patients $(p=0.001)$. Additionally, in the Montreal cohort, H. pylori seropositivity was found to be associated with an apparent decrease of NSCLC patient progression-free survival on anti-PD-1 therapy.

Conclusion Our study unveils for the first time that the stomach microbiota affects the response to cancer immunotherapies and that $H$. pylori serology would be a powerful tool to personalize cancer immunotherapy treatment.

\section{INTRODUCTION}

Immune checkpoint inhibitors (ICIs) aim to reinvigorate the immune system toward cancer cells. However, despite unprecedented results, the majority of patients do not respond to ICIs and several individualistic factors have been associated

\section{Significance of this study}

What is already known on this subject?

- Previous preclinical and clinical studies demonstrated that the large and small intestinal microbiota affect the efficacy of cancer immunotherapies.

What are the new findings?

- Our study unveils for the first time that the stomach microbiota of Helicobacter pylori infected hosts affects the response to cancer immunotherapies.

How might it impact on clinical practice in the foreseeable future?

- H. pylori serology would be a powerful tool to personalise cancer immunotherapy treatment.

with resistance such as the composition of the gut microbiota and tumour immune contexture. ${ }^{1}$

Vétizou $e t a l^{2}$ demonstrated that the antitumour effects elicited by anticytotoxic T-lymphocyteassociated protein 4 (CTLA4) blockade in mice were dependent on the presence of Bacteroides fragilis, Bacteroides thetaiotaomicron and Burkholderiales in the gut microbiota. Other research groups have identified additional immune-potentiating bacteria in the gut such as Akkermansia muciniphila, Faecalibacterium prausnitzii and Bifidobacterium species present in those who respond to immunotherapy compared with those who do not. ${ }^{34}$ More importantly, human data clearly demonstrated that the composition of the intestinal microbiome had a considerable influence on the effectiveness of anticancer immunotherapies $^{3-5}$ and that the use of antibiotics compromised the efficacy of programmed cell death protein 1 (PD-1) blockade therapy. ${ }^{6}$ Paradoxically, no common bacterial taxa were clearly identified as key indicators and/or modulators of ICI responses across published studies. ${ }^{1}$ This may be due to technical issues linked to microbiome analysis or to geographical factors such as diet and lifestyle. Another possibility is that the analysis of the patient's faecal samples solely may be overlooking key information. Indeed, the small intestine ${ }^{7}$ and/ or stomach microbiota may also profoundly affect responses to ICIs. 
Helicobacter pylori (H. pylori), which colonises the stomach mucosa of $50 \%$ of the world population, actively manipulates host tissues to establish an immunosuppressive environment that maintains chronic infection. ${ }^{8} \mathrm{H}$. pylori dampens the effector functions of $\mathrm{CD}^{+}{ }^{+} \mathrm{T}$ cells, ${ }^{8}$ dendritic cells (DCs) ${ }^{9-12}$ and macrophages ${ }^{8}$ and promotes the generation of regulatory $\mathrm{T}$ cells (Tregs) ${ }^{10}$ and myeloid-derived suppressor cells. ${ }^{13}$ Previous observations demonstrated that systemic inflammatory disorders such as asthma, lupus, inflammatory bowel disease and eosinophilic esophagitis are negatively associated with $H$. pylori infection in the human population ${ }^{14-17}$ and in animal models. ${ }^{18-21}$ This suggests that $H$. pylori may mitigate unbalanced systemic immune responses. The negative impact of $H$. pylori-mediated immunomodulation on a large number of immune cell types associated with antitumour immunity allows for the important consideration that $H$. pylori infection may decrease the response to cancer immunotherapies.

\section{RESULTS \\ H. pylori infection decreases the effectiveness of cancer immunotherapies in preclinical models}

In order to test whether $H$. pylori infection diminishes the effectiveness of ICIs, we first evaluated whether H. pylori decreases the efficacy of anti-CTLA4 therapy using an MC38 colon adenocarcinoma model (see online supplemental figures S1A). Interestingly, the tumour volumes of non-infected mice undergoing anti-CTLA4 treatment were significantly smaller than those of H. pylori-infected mice (figure 1A). We next evaluated whether H. pylori decreases the efficacy of anti-CTLA4/programmed death ligand 1 (PD-L1) combination therapy ${ }^{22}$ (see online supplemental figures $\mathrm{S} 1 \mathrm{~B}$ ). As shown in figure $1 \mathrm{~B}$, the tumour volumes of non-infected mice undergoing anti-CTLA4/PD-L1 treatment were significantly smaller than those of infected mice. These results demonstrate that $H$. pylori-infected mice are less responsive to CTLA4 blockade alone or in combination with anti-PD-L1. Furthermore, by using a B16-OVA melanoma model, we evaluated whether $H$. pylori decreases the efficacy of a vaccine-based cancer immunotherapy. Tumour-bearing mice were transferred with OVA-specific CD ${ }^{+} \mathrm{T}$ cells (OT-1 cells) and were immunised with OVA peptide (SIINFEKL) emulsified in $\mathrm{CpG}^{23}$ (see online supplemental figures S1C). Remarkably, the tumour volumes of vaccinated non-infected mice were significantly smaller than those of vaccinated infected mice (figure 1C), demonstrating that $H$. pylori-infected mice are less responsive to anti-cancer vaccination. In addition, we observed that the infection of the stomach mucosa with Helicobacter felis $(H$. felis), a Helicobacter species lacking a number of the virulence factors of $H$. pylori such as Cag pathogenicity island and vacuolating cytotoxin (VacA), ${ }^{24}$ does not decrease the effectiveness of anti-cancer vaccination (figure 1D) or anti-CTLA4 therapy (figure 1E). Lastly, we evaluated the effect of $H$. pylori infection on the effectiveness of immunotherapy in tumours developing in situ. We selected the model of azoxymethane (AOM)/dextran sodium sulfate (DSS) colon cancer, as it has recently been shown that anti-CTLA4 therapy reduces the tumour burden in this model (see online supplemental figures S1D). ${ }^{25}$ Remarkably, the number of colon tumours in non-infected mice undergoing anti-CTLA4 treatment was significantly lower than those of H. pylori-infected mice (figure $1 \mathrm{~F}$ ). Collectively, we provide evidence demonstrating that the presence of $H$. pylori in the gastric microbiota specifically jeopardises the efficacy of cancer immunotherapies.

\section{H. pylori-mediated immunosuppression of cancer immunotherapies is independent of the $H$. pylori-induced modification of the faecal microbiota}

We investigated whether $H$. pylori-mediated immunosuppression relies on the modulation of the composition of the gut microbiota. To evaluate the role of the gut microbiota in $H$. pylori-induced immunosuppression, we performed three types of experiments. As a first approach, we performed co-housing experiments. Due to the coprophagia behaviour of mice, the composition of the faecal microbiota of co-housed mice is very similar. However, co-housing does not allow for H. pylori transmission from infected to non-infected neonate/adult mice (see online supplemental figures S2A). Using a B16-OVA melanoma model, we observed that the tumour volumes of vaccinated non-infected mice were significantly smaller than those of vaccinated infected mice (figure 2A). Similarly, infected mice were less responsive to anti-CTLA4 therapy in the MC38 model (data not shown). Next, we performed $16 \mathrm{~S}$ rRNA gene sequencing of the intestinal microbiota both before and during vaccine-based immunotherapy administration (see online supplemental figures S1C). We observed that the intestinal microbiota of non-infected and infected mice differed at steady state (figure $2 \mathrm{~B}$ ), confirming the work of Kienesberger et al. ${ }^{26}$ Notably, we found that H. pylori infection leads to a decreased bacterial colonisation of Lachnospiraceae and Erysipelotrichaceae genera and to an increased bacterial colonisation of the Bifidobacterium genus (figure 2C). Infected mice displayed higher alpha diversity compared with non-infected mice. Very interestingly, similarly increased alpha diversity has been described in $H$. pylori-infected individuals compared with matched H. pylori-negative controls. ${ }^{27}$ Paradoxically, high alpha diversity and high prevalence of Bifidobacterium are commonly observed in mice which develop a favourable anticancer immune response following immunotherapy administration. ${ }^{128}$ Interestingly, the faecal microbiota of non-infected and infected mice were similar in the vaccine-based immunotherapy setting (figure 2D). Lastly, we transplanted faeces from $H$. pyloriinfected mice to non-infected mice and performed cancer immunotherapy using the B16-OVA melanoma model (see online supplemental figures S1E). We observed that the vaccinated noninfected mice, transplanted with faeces of infected mice, maintained the ability to efficiently control tumour growth compared with non-vaccinated counterparts (figure 2E). Collectively, co-housing experiments, 16S rRNA gene sequencing and faecal transplantation demonstrate that $H$. pylori-mediated immunosuppression of cancer immunotherapies is independent of the $H$. pylori-induced modification of the faecal microbiota.

\section{Eradication of $H$. pylori infection by antibiotherapy does not increase the efficacy of vaccine-based immunotherapy} We evaluated whether the eradication of $H$. pylori infection by antibiotherapy reverts the $H$. pylori-induced decreased efficacy of cancer immunotherapies. Antibiotic administration does not substantially rescue the efficacy of vaccine-based cancer immunotherapy in infected mice (figure $2 \mathrm{~F}$ ). Together with the known detrimental effect of antibiotic administration on the efficacy of immunotherapies in cancer patients, ${ }^{6}{ }^{29}$ this result suggests that antibiotic administration to cure $H$. pylori infection is most likely a poor option to increase the efficacy of cancer immunotherapies in patients.

\section{H. pylori jeopardises tumour specific immune responses}

We next studied the impact of $H$. pylori on the functionality of the immune system. Using flow cytometric analysis, we analysed 
A
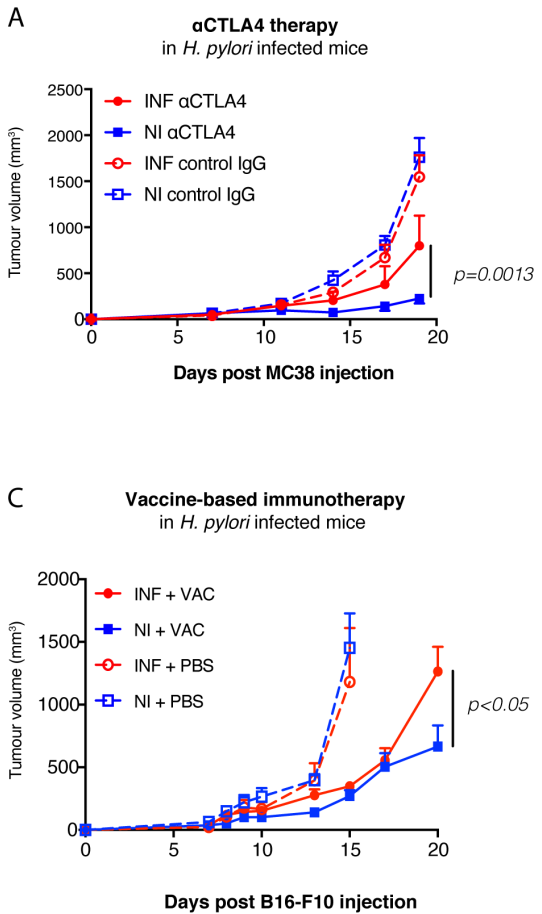

E

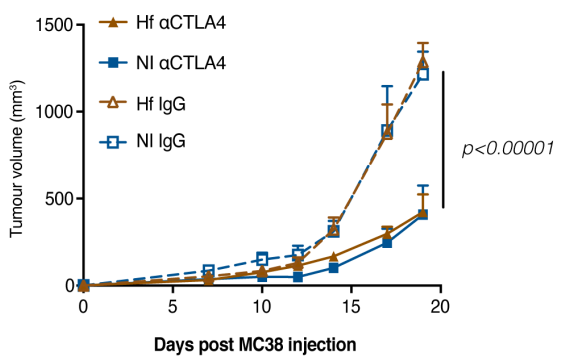

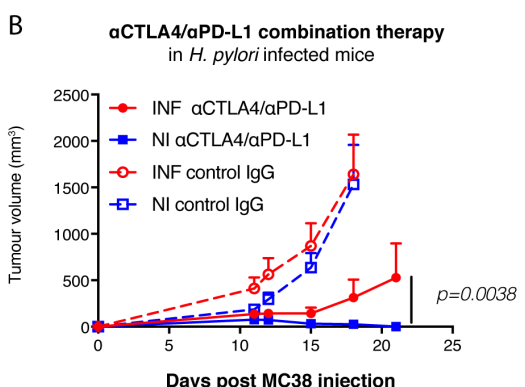

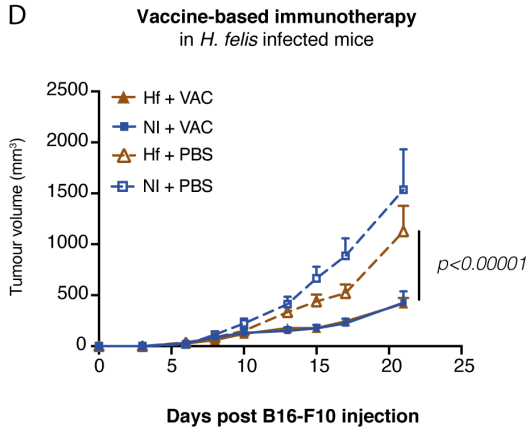

$\mathrm{F}$ aCTLA4 therapy of colon cancer in H. pylori infected mice



Figure 1 Helicobacter pylori infection decreases the effectiveness of cancer immunotherapies in preclinical models. (A) Mice were injected with MC38 colon adenocarcinoma cells and intraperitoneally injected with anti-CTLA4 ( $\alpha$ CTLA4) or IgG2b isotype as control. At day 19, tumour volumes of non-infected (NI) and infected (INF) mice treated with anti-CTLA4 therapy were significantly different $(p<0.01$, two-way analysis of variance (ANOVA)). Experimental groups included seven mice. (B) MC38 tumour growth kinetics of NI and INF mice treated with anti-CTLA4/PD-L1 ( $\alpha$ CTLA4/ $\alpha P D-L 1)$ combination therapy. Anti-CTLA4/PD-L1 treatment resulted in tumour rejection in seven of eight NI mice, whereas anti-CTLA4/PD-L1-treated INF mice showed tumour rejection in two of six mice. At day 21, the tumour volumes of NI and INF mice treated with anti-CTLA4/PD-L1 antibodies were statistically different ( $p=0.009$, two-way ANOVA). Experimental groups included five to eight mice. (C) B16-OVA tumour growth kinetics of NI and INF mice treated with an anti-cancer vaccine (VAC) or phosphate-buffered saline (PBS) as a control. Vaccination resulted in decreased tumour growth in NI mice, however, did not efficiently limit tumour growth in INF mice. At day 20, tumour volumes of vaccinated NI and INF mice were statistically different ( $<<0.05$, two-way ANOVA). Experimental groups included five to eight mice. (D) B16-OVA tumour growth kinetics of $\mathrm{NI}$ and $\mathrm{H}$. felis (H.f)-infected mice treated with an anti-cancer vaccine or PBS as a control. Vaccination resulted in statistically significant decrease in tumour growth in $\mathrm{NI}$ and H.f-infected mice ( $\mathrm{p}<0.001$, two-way ANOVA). Experimental groups included nine to ten mice. (E) MC38 tumour growth kinetics of $\mathrm{NI}$ and H.f-infected mice treated with anti-CTLA4 ( $\alpha$ CTLA4) therapy. Anti-CTLA4 treatment resulted in decreased tumour growth in both NI and $H . f$ mice. At day 19, the tumour volumes of $\mathrm{NI}$ and $H . f$ mice treated with anti-CTLA4 antibodies were not statistically different ( $p=0.65$, two-way ANOVA). Experimental groups included six mice. (F) Number of colon tumours of $\mathrm{NI}$ and $H$. pylori INF-infected mice treated with anti-CTLA4 ( $\alpha \mathrm{CTLA} 4)$ or IgG2b isotype as control. Anti-CTLA4 injection resulted in decreased tumour number in NI mice but not in INF mice. At sacrifice, tumour numbers of anti-CTLA4-injected NI and INF mice were statistically different ( $<<0.0013$, Mann-Whitney test). Experimental groups included ten mice. For the experiments described in figure $1 \mathrm{~A}-\mathrm{F}$, the infectious status of each individual mouse was confirmed at sacrifice by performing rapid urease tests and/or colony forming units on the stomach (see online supplemental figures S2A-F). CTLA4, cytotoxic T-lymphocyte-associated protein 4; PD-L1, programmed death ligand 1.

the absolute cell number and activation states of $\mathrm{CD}^{+}$and $\mathrm{CD}^{+} \mathrm{T}$ cells, Tregs, migratory and resident DC1 cells, DC2 cells, monocyte-derived DCs, macrophages and monocytes in the spleen and lymph nodes of non-infected and infected mice at steady state. We also analysed $\mathrm{T}$ cell subsets and monocytes in the blood. We identified that H. pylori infection does not impact the amount nor the activation states of the aforementioned cell types (see online supplemental figures S3 and S4). 
A



c

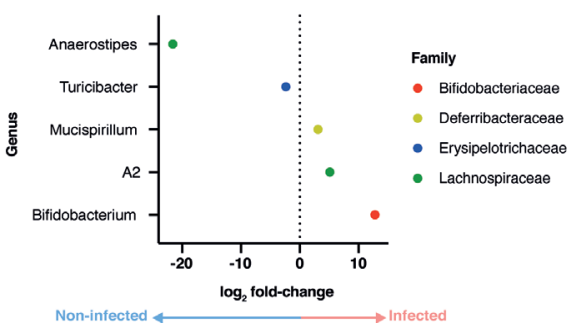

B

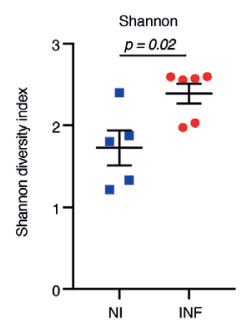

NMSD - Bray-Curtis
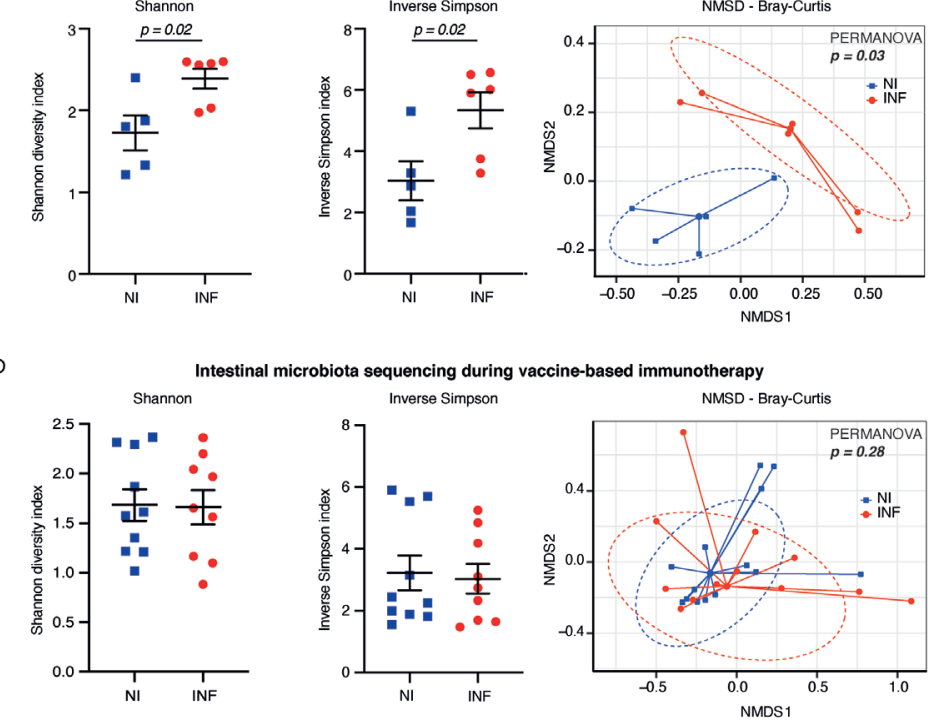


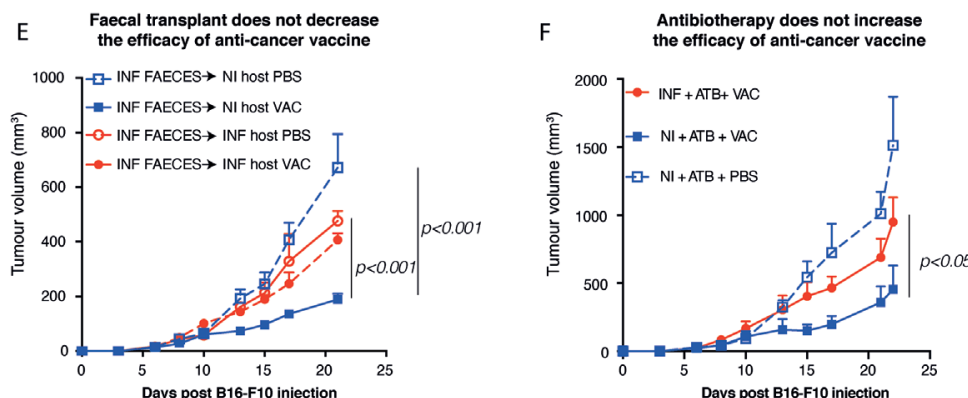

Figure 2 Helicobacter pylori-mediated immunosuppression of cancer immunotherapies is independent of the $\mathrm{H}$. pylori-induced modification of the faecal microbiota. (A) Co-housing experiments, non-infected (NI) $(n=9)$ and H. pylori-infected (INF) ( $n=10)$ mice were subcutaneously injected with B16-OVA tumour cells and vaccinated. Vaccination (VAC) showed very limited tumour growth inhibition in INF mice. At days 27 and 30, the tumour volumes of vaccinated $\mathrm{NI}$ and INF mice were statistically different $(\mathrm{p}<0.001$, two-way analysis of variance (ANOVA)). At sacrifice, we performed rapid urease tests on the stomach of $\mathrm{NI}$ and INF co-housed mice. We only detected $H$. pylori infection in INF and not in NI mice, confirming that co-housing does not allow for H. pylori transmission (see online supplemental figures S2A). (B) Shannon, Inverse Simpson and Bray-Curtis based Non-metric Multidimensional Scaling (NMDS) analysis of 16S rRNA gene sequencing of the intestinal microbiota before the initiation of the vaccinebased immunotherapy. 165 rRNA analyses were performed in non-co-housing conditions. At steady state, INF mice displayed higher alpha diversity compared with NI mice ( $p=0.02$, Mann-Whitney test). (C) $16 \mathrm{~S}$ rRNA gene sequencing of the intestinal microbiota before the initiation of the vaccinebased immunotherapy. Differential abundance analysis performed at the genus taxonomic level comparing 165 rRNA sequencing of INF vs NI mice at steady state. Only bacteria with an adjusted $p$ value $<0.05$ are displayed (Benjamini-Hochberg method). (D) Shannon, Inverse Simpson and BrayCurtis based Non-metric Multidimensional Scaling (NMDS) analyses of 165 rRNA gene sequencing of the intestinal microbiota during vaccine-based immunotherapy. The faecal microbiota of INF and NI mice were similar on day 8 post $\mathrm{CpG} / \mathrm{OVA}$ vaccination (no statistical difference, Mann-Whitney test). (E) Faecal transplantation of INF faeces into NI or INF recipient mice. Recipient mice were treated with antibiotics and orally administered with $100 \mathrm{mg}$ of faeces from INF mice on a daily basis for 5 days. Two weeks after the last oral gavage, vaccine-based immunotherapy was initiated on B16OVA tumour-bearing mice (online supplemental figures S1E). Remarkably, vaccinated NI mice transplanted with INF faeces $(n=9)$ are still capable to very efficiently control tumour growth compared with non-vaccinated (PBS) counterparts $(n=7)(p<0.001$, two-way ANOVA). Additionally, vaccinated $\mathrm{NI}$ mice transplanted with INF faeces $(\mathrm{n}=9)$ show better tumour growth control compared with vaccinated INF mice transplanted with INF faeces $(n=4)(p<0.001$, two-way ANOVA). We observed that faecal transplantation of INF faeces to NI recipient mice do not allow for $H$. pylori infection (online supplemental figures S2C). (F) Antibiotherapy (ATB) does not increase the efficacy of anti-cancer vaccination. Mice were infected with $H$. pylori during the neonatal period and treated with ATB at 6 weeks of age. One-month post ATB, mice were engrafted with B16-OVA melanoma cells and vaccinated (online supplemental figures S1F). We observed that the eradication of $H$. pylori infection by ATB (online supplemental figure S2B) did not substantially increase the efficacy of cancer vaccination ( $p<0.05$, two-way ANOVA). For panels $A-D$ and $F$, data shown are representative of two independent experiments. For the experiments described in figure $2 A-D$, the infectious status of each individual mouse was confirmed at sacrifice by performing rapid urease tests and/or colony forming unit on the stomach (see online supplemental figures S2A-C).

Identifying no impact of $H$. pylori on the immune cells at steady state, we next evaluated whether $H$. pylori affects the previously described immune cells in the context of cancer. We observed similar absolute numbers of immune cells in the blood, spleen, non-draining and tumour-draining lymph nodes (ndLNs and
tdLNs, respectively) and tumour(s) of non-infected and infected mice (see online supplemental figure S5). Importantly, flow cytometric analysis of immune cells isolated from the aforementioned organs/tissue/tumour(s) of MC38 tumour-bearing mice showed that the presence of $H$. pylori alone is unable to substantially 
A tolns (number of cells)
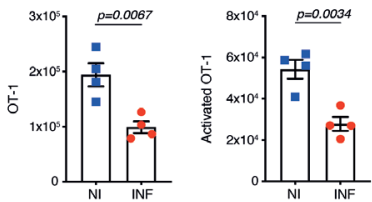

ndLNs (number of cells)
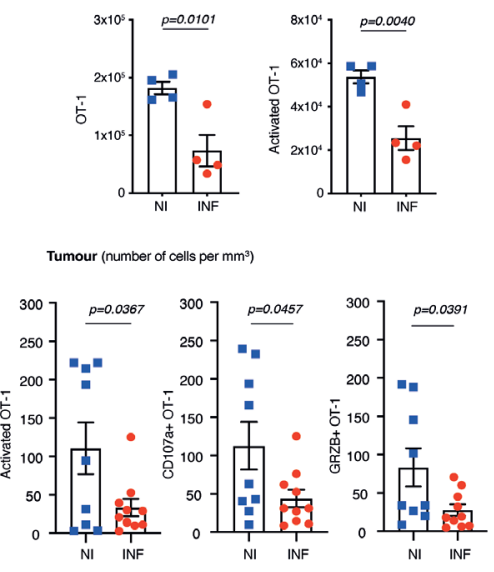

B Vaccine-induced proliferation and cytotoxic
activites of OT-1cells
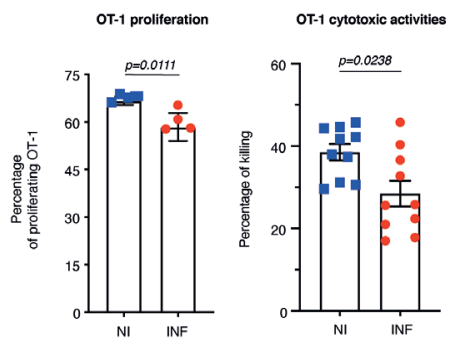

C

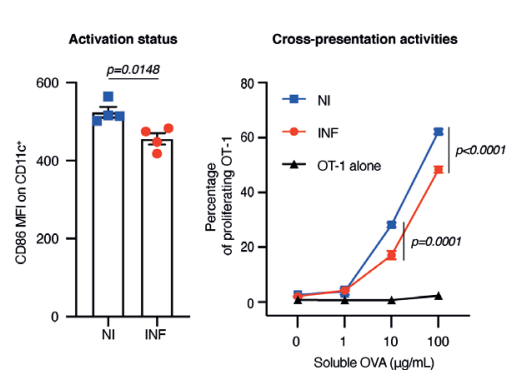

D The presence of $H$. pylori affects innate immune responses


Figure 3 Helicobacter pylori jeopardises tumour-specific immune responses. (A) Absolute cell number and activation status of OT-1 CD8 ${ }^{+} T$ cells isolated from the tdLN, ndLN and tumour(s) of non-infected (NI) and infected (INF) vaccinated B16-OVA tumour-bearing mice (days 10 and 15 post B16 inoculation, respectively, for LNs and tumours). Activated OT-1 cells that were defined has $C D 44^{+} C D 62 L^{-}$. (B) Left panel: percentage of proliferating OT-1 cells in the tdLN of NI and INF mice evaluated by carboxyfluorescein succinimidyl ester staining. Right panel: in vivo cytotoxic activities of OT-1 cells in vaccinated NI and INF mice. (C) The presence of $H$. pylori affects the functionality of DCs. Left panel: expression of the activation marker CD86 on CD11 $\mathrm{c}^{+} \mathrm{CD} 11 \mathrm{~b}^{-} \mathrm{CD} 8 \mathrm{a}^{+} \mathrm{DCs}$ in the tdLN of NI and INF mice. Right panel: frequency of proliferating OT-1 cells on ex vivo stimulation with DCs isolated from the spleen of NI or INF mice. Data are representative of three independent experiments $(N I, n=9 ; I N F, n=10)$ $(p<0.05$, unpaired t-test). (D) The presence of $H$. pylori affects innate immune responses, as determined by inflammatory cytokine (TNF $\alpha$, IFN $\gamma$, IL-6 and IL-17) levels in the serum of $\mathrm{NI}$ and INF mice. NI and INF B16-OVA tumour-bearing mice were injected with OT-1 cells and vaccinated with OVA in CpG. The serums were recovered on days 1,2 and 3 postvaccination. For panels $A-C$, data shown are representative of three independent experiments. For panel $D$, data shown are representative of two independent experiments. For the experiments described in figure 3A-D, the infectious status of each individual mouse was confirmed at sacrifice by performing rapid urease tests and/or colony forming unit on the stomach (see online supplemental figures S2E). DCs, dendritic cells; MFI, mean fluorescence intensity; ndLN, non-draining lymph nodes; tdLN, tumour-draining lymph node.

affect the immune cell populations associated with antitumour immunity (see online supplemental figures S6 and S7). Lastly, we analysed the immune cells in MC38 tumour-bearing mice undergoing anti-CTLA4 treatment. We observed similar numbers of tumour infiltrating immune cells between non-infected and infected mice (see online supplemental figure S8). In addition, absolute cell numbers and activation status of $\mathrm{CD} 4^{+}$and $\mathrm{CD} 8^{+}$ $\mathrm{T}$ cells in the tumours, tdLNs, ndLNs and blood were similar in non-infected and infected mice (see online supplemental figure S8 and data not shown). It is noteworthy to indicate that we observed a substantial reduction in the number of myeloid cells, notably, a tendency of reduction in the number of activated monocytes, in the tumours of infected mice compared with noninfected mice undergoing anti-CTLA4 treatment (see online supplemental figure S8). These results are reminiscent of the work of Iida $\mathrm{et} \mathrm{al}^{30}$ showing that the gut microbiota can modulate the infiltration of monocyte-derived cells into the tumours, impacting the response to cancer therapy.

The efficacy of cancer immunotherapies relies on the generation of tumour-specific immune responses. We next evaluated whether $H$. pylori jeopardises tumour-specific immune responses, first by studying the absolute cell number and differentiation status alongside the effector functions of OT- 1 cells extracted from the tumour(s), ndLNs and tdLNs of vaccinated B16-OVA tumour-bearing mice. We observed a decreased number and activation status of tumour-specific $\mathrm{T}$ cells in the ndLNs and tdLNs of infected mice (figure 3A). The decreased number of OT-1 cells in LNs is in part due to a lower vaccine-induced proliferation of OT- 1 cells observed in the LN draining the vaccination site in infected mice compared with their non-infected counterparts (figure 3B). Moreover, we observed a decreased number of activated OT- 1 cells in the tumours of infected mice (figure 3A). Remarkably, a similar number and activation status of OT- 1 cells were found in tumour and tdLNs of $\mathrm{H}$. felis-infected or noninfected mice (see online supplemental figure S9A). Lastly, by performing in vivo killing assay, we observed that the cytotoxic activities of OT-1 cells generated by vaccination in $H$. pyloriinfected mice were lower than those generated in non-infected or $\mathrm{H}$. felis-infected mice (see figure 3B and online supplemental figure S9B). Collectively, our data show that vaccine-primed tumour-specific immune responses are specifically reduced in $H$. pylori-infected mice.

It has previously been described that the gut microbiota can modulate DC function, which impacts $\mathrm{CD} 8^{+} \mathrm{T}$ cell priming. ${ }^{28} 31$ With this, we reasoned that the reduction of tumour immune responses may be associated with $H$. pylori-induced DC defects. ${ }^{9-12}$ We indeed detected defects in activation of DC1 and DC2 cells in the tdLNs of infected mice in as little as 24 hours post B16-OVA engraftment (figure 3C). Next, by performing an in vitro co-culture assay, we observed that splenic DCs of 
infected mice promote lower antigen-specific proliferation of OT-1 cells (figure 3C). Taken together, these results demonstrate that $H$. pylori decreases DC activation processes, ${ }^{12} 32$ their cross-presentation activities and jeopardises the tumour-specific immune responses initiated by vaccination.

Lastly, we observed a significant decrease of inflammatory cytokine production in the serum of infected mice on day 1 post OVA/CpG vaccination (figure $3 \mathrm{D}$ ). Notably, IFN $\gamma$, a cytokine playing a pivotal role in the efficacy of tumour vaccination (see online supplemental figure S10), was drastically reduced in the serum of infected mice (figure 3D). Altogether, it can be concluded that by dampening innate immune responses (DCs cross-presentation activities and vaccine-induced inflammatory cytokine production), H. pylori decreases the tumour-specific immune responses initiated by vaccine-based immunotherapy, leading to a decrease in its efficacy.

\section{H. pylori seropositivity is associated with reduced effectiveness of anti-PD1 immunotherapy in patients with NSCLC}

We performed a retrospective study in two independent cohorts of patients with non-small cell lung cancer (NSCLC) to evaluate the correlation between $H$. pylori seropositivity and the effectiveness of cancer immunotherapies. H. pylori seropositivity was determined using validated commercial ELISA tests. In the first cohort of 60 patients with NSCLC from Dijon (France), we detected $H$. pylori antigen-directed IgG antibodies in the serum of 18 patients. In the second cohort of 29 patients with NSCLC from Montreal (Canada), we detected H. pylori antigen-directed IgG antibodies in the serum of 8 patients. Remarkably, in the Dijon cohort, H. pylori seropositivity was found to be associated with a clearly defined decrease of NSCLC patient survival on anti-PD-1 therapy (figure $4 \mathrm{~A}, \mathrm{p}=0.001$ ). Survival medians were 6.7 months for $H$. pylori seropositive patients vs 15.4 months for seronegative patients. Additionally, in the Montreal cohort, $H$. pylori seropositivity was found to be associated with an apparent decrease of NSCLC patient progression-free survival on anti$\mathrm{PD}-1$ therapy (figure $4 \mathrm{~B}, \mathrm{p}<0.05$ ) and a numerical difference for overall survival $(9.3$ months for $H$. pylori seropositive patients compared with 21.7 months for seronegative patients). These data obtained from two independent cohorts of patients with NSCLC on anti-PD-1 therapy clearly demonstrate that $H$. pylori seropositivity is associated with lower effectiveness of anti-PD-1 immunotherapy. Additional prospective studies will further validate our data. Lastly, we performed RNAseq analysis of RNA extracted from formalin-fixed paraffin-embedded tumours from
A Dijon cohort

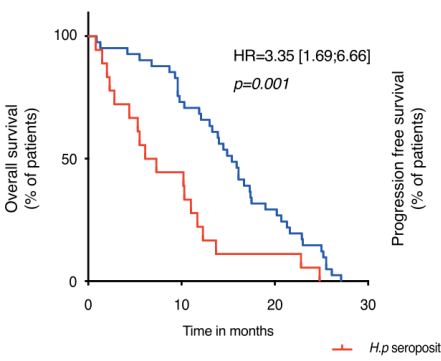

- H.p seropositive
$+\quad$ H.p seronegative

C RNAseq analysis

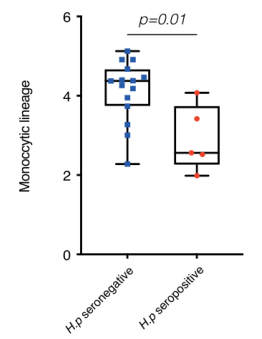

B

Montreal cohort
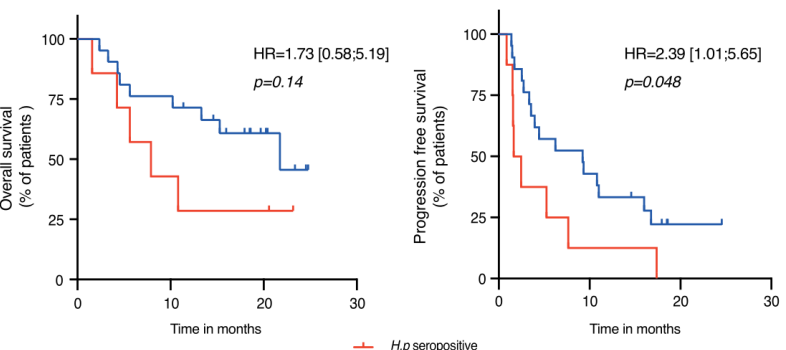

$\perp \quad$ H.p seropositive
$+\quad$ H.p seronegative

Figure 4 Helicobacter pylori seropositivity is associated with reduced effectiveness of anti-PD-1 immunotherapy in patients with non-small cell lung cancer (NSCLC). (A) Dijon cohort: overall survival and overall progression-free survival of patients with NSCLC from the Dijon cohort (60 patients). Kaplan-Meier curves depicting the overall survival of patients with NSCLC treated with nivolumab or pembrolizumab (anti-PD-1 monoclonal antibodies). H. pylori seropositivity was found to be associated with a clearly defined decrease of NSCLC patient survival on anti-PD-1 therapy ( $p=0.001$, Wald test as part of a univariate of Cox model). (B) Montreal cohort: overall survival and overall progression-free survival of patients with NSCLC from the Montreal cohort (29 patients). Kaplan-Meier curves depicting the overall survival of patients with NSCLC treated with nivolumab or pembrolizumab. Log-rank (Mantel-Cox) test ${ }^{*} p<0.05$. (C) H. pylori infection substantially affects myeloid cells in the tumour(s) of patients with NSCLC. RNAseq analysis was performed on the tumours of patients from the discovery cohort. MCP-counter software was used to determine the tumour infiltration of $\mathrm{CD}^{+} \mathrm{T}$ cells, $\mathrm{CD} 8^{+} \mathrm{T}$ cells, cytotoxic lymphocytes, NK cells, B lymphocytes, cells originating from monocytes (monocytic lineage), myeloid-derived dendritic cells, neutrophils, endothelial cells and fibroblasts. We observed that in the tumours of $H$. pylori seronegative patients, there was a substantially higher expression of genes expressed by cells originating from monocytes compared with $H$. pylori seropositive patients $(p=0.01$, Wilcoxon test). (D) Gene-set enrichment analyses (GSEA) were performed on the RNAseq data and revealed that $H$. pylori infection substantially decreases the expression of genes that are controlled by type I IFN, IFN $\gamma$ and IL-6 in the tumours of patients with NSCLC undergoing immunotherapy. PD-1, programmed cell death protein 1. 
patients with NSCLC from the Dijon cohort. ${ }^{33}$ Although we analysed a limited number of patients, we observed a significantly decreased number of cells from the monocyte lineage (figure 4C) and a substantially decreased expression of genes induced by type I interferon, IFN $\gamma$ and IL-6 in the tumours of infected patients compared with non-infected patients (figure 4D). These results are reminiscent of the preclinical data (figure 3D and see online supplemental figure S8C1), suggesting that the dampening of the innate immune responses by $\mathrm{H}$. pylori in infected hosts may decrease the efficacy of cancer immunotherapies. ${ }^{30}$

\section{DISCUSSION}

In this study, we show that $H$. pylori infection partially blocks the activity of ICIs and vaccine-based cancer immunotherapy in murine models. Mechanistically, we observed that $H$. pylori dampens the innate and adaptive immune responses of infected hosts and, more specifically, inhibits the antitumoural $\mathrm{CD} 8^{+}$ $\mathrm{T}$ cell responses by altering the cross-presentation activities of DCs. In addition, we report that $H$. pylori seropositivity is associated with a reduced effectiveness of anti-PD1 immunotherapy in patients with NSCLC.

It has already been reported that $H$. pylori-infected mice have defective DC activation processes. ${ }^{9-12}$ These DC defects were observed in vivo within the gastric mucosa, mesenteric lymph nodes as well as in the lungs of asthmatic mice. ${ }^{12}$ Remarkably, this $H$. pylori-induced DC hypoactivation leads to decreased activation of allergen-specific Th2 cells in asthmatic mice. ${ }^{19} 3435$ In this study, we provide evidence that the $H$. pylori-induced DC defects also lead to decreased activation of tumour-specific CD8 ${ }^{+} \mathrm{T}$ cells. $H$. pylori infection not only affects DC function but also that of monocytes and/or macrophages. Indeed, in humans, we observed a decreased number of cells from the monocyte lineage and a substantially decreased expression of genes induced by type I interferon, IFN $\gamma$ and IL- 6 in the tumours of infected patients with NSCLC undergoing anti-PD1 treatment (figure 4C). In addition, we observed a tendency of reduction in the number of activated monocytes in the tumours of infected mice undergoing anti-CTLA4 treatment (online supplemental figure S8) and a blunted production of inflammatory cytokines in the serum of vaccinated infected mice (figure 3D). Different virulence factors produced by $H$. pylori such as VacA, $\gamma$-glutamyl transpeptidase, neutrophil-activating protein of $H$. pylori and urease have been described to impair myeloid cell activities. ${ }^{36}$ Additional experiments will determine whether those H. pyloriderived factors are key in decreasing the effectiveness of cancer immunotherapies.

An informative and puzzling finding of our study is that the eradication of $H$. pylori infection by antibiotherapy does not revert the $H$. pylori-induced hyporesponsiveness to cancer immunotherapy. This could be a consequence of the non-specific antibiotherapy-mediated reduction of immune-potentiating bacteria and/or $H$. pylori-induced neonatal imprinting ${ }^{37}$ of the immune system that persists even after its eradication. Such neonatal imprinting that persistently modulates the functioning of the immune system has already been reported in mice. ${ }^{38} 39$ Thus, the eradication of $H$. pylori using a strategy that spares the microbiota, such as vaccination, would likely prove to be unsuccessful, as the immune system would have been previously imprinted.

Our clinical study was performed in two independent centres, one located in Europe and the other in North America. Results from both cohorts clearly show that the efficacy of PD- 1 immunotherapies is lower in $H$. pylori seropositive patients with NSCLC.
In the perspective that this result is confirmed in prospective studies, it has two important clinical implications. First, serological tests detecting $H$. pylori seropositivity may be a powerful tool in predicting the efficacy of ICIs for patients with NSCLC cancer. Future clinical studies will establish whether the same correlation between $H$. pylori seropositivity and the reduced effectiveness of cancer immunotherapies can be extended to patients suffering from other forms of cancer. Second, it may be very useful to consider $H$. pylori seropositivity as a confounding factor that may prevent the identification of bacterial taxa, in patient's faecal samples, affecting positively or negatively, the response to ICIs. Similarly, $H$. pylori seropositivity might also preclude any clinical benefits brought forth by faecal microbiota transplantation in ICI-treated patients. ${ }^{4-42}$

In summary, our study is in alignment with the recognised major role played by the microbiome on the efficacy of cancer immunotherapies. ${ }^{1}$ However, our study unveils for the first time that the stomach microbiota affects the response to ICIs and that H. pylori serology would be a powerful tool to personalise the treatment of patients in the context of cancer immunotherapies.

\section{MATERIALS AND METHODS \\ Mice}

CD $45.2^{+}$C57BL/6 mice were obtained from Charles River (Ecully, France), while CD45.1 $1^{+}$OT-1 C57BL/6 mice were kindly provided by Dr Gregory Verdeil (University of Lausanne). Mice were bred under specific pathogen-free conditions. All animal experiments were performed in accordance with cantonal laws of animal protection.

\section{H. pylori infection}

$H$. pylori $\mathrm{P} 49$, a human clinical isolate adapted to mice and $H$. felis strain ATCC $49179^{43}$ were grown in brain heart infusion (BHI) supplemented with $10 \%$ fetal bovine serum (FBS) in microaerophilic conditions. Mice were infected twice during the neonatal stage (4 and 5 days of age) with $2.5 \times 10^{8} \mathrm{H}$. pylori $\mathrm{P} 49$ bacteria or once with $5 \times 10^{7} \mathrm{H}$. felis ( 5 days of age). Bacteria were administered orally in $20 \mu \mathrm{L}$ of BHI. The control group received $20 \mu \mathrm{L}$ of $\mathrm{BHI}$. For co-housing experiments, neonates belonging to the same litter were infected with $H$. pylori or treated with BHI as control and then weaned in the same cage. Infected and non-infected littermates received the same therapy.

\section{Assessment of $H$. pylori colonisation}

Rapid urease test (Cleartest Servoprax, Germany) and quantification of $H$. pylori colony forming units were used to assess infection status as previously described. ${ }^{44}$

\section{MC38 tumour cells}

Prof Greta Guarda (Biochemistry Institute, Lausanne, Switzerland) kindly provided MC38 colon adenocarcinoma cells. The cells were cultured in Roswell Park Memorial Institute (RPMI) medium supplemented with $10 \% \mathrm{FBS}$ and $1 \%$ penicillin/streptomycin at $37^{\circ} \mathrm{C}, 5 \% \mathrm{CO}_{2}$.

\section{B16-0VA tumour cells}

B16-F10 mouse melanoma cells expressing OVA protein (B16OVA) were kindly provided by Dr Gregory Verdeil. ${ }^{23}$ The cells were cultured in RPMI supplemented with $10 \%$ FBS, $1 \%$ penicillin/streptomycin, $1 \mathrm{mM}$ sodium pyruvate, $10 \mu \mathrm{g} / \mathrm{mL}$ G418 at $37^{\circ} \mathrm{C}, 5 \% \mathrm{CO}_{2}$. 


\section{MC38 tumour model and ICI treatments}

MC38 tumour cells $\left(5 \times 10^{5}\right)$ were injected subcutaneously into the shaved flank skin of adult male CD $45.2^{+}$mice neonatally infected with $\mathrm{H}$. pylori or treated with BHI as control. Mice received in $100 \mu \mathrm{L}$ PBS, either IgG2b isotype (clone MPC-11, BioXCell) or anti-mouse anti-CTLA4 (clone 9D9, BioXCell) alone or in combination with anti-mouse anti-PD-L1 (clone 10F.9G2, BioXCell) on days 7, 10, 13 and 16 post tumour engraftment. We injected intraperitoneally $200 \mu \mathrm{g}$ of antibody for the first injection and $100 \mu \mathrm{g}$ for the following three injections. Tumour volumes were measured according to length and width using Vernier callipers from day 7 following tumour engraftment and every 2-3 days thereafter (see online supplemental figures S1A, B).

\section{AOM/DSS colon cancer model and ICl treatments}

Infected and non-infected C57BL/6 mice were intraperitoneally injected with AOM at 8 weeks of age. ${ }^{45}$ Mice were orally administered with three rounds of DSS. Ten days after the last administration of DSS, mice were injected with $100 \mu \mathrm{g}$ anti-CTLA4 antibodies (five injections, 3 days apart) and sacrificed 7 days after the last anti-CTLA4 injection (see online supplemental figures S1D). At sacrifice, we numerated the number of tumours within the colon. ${ }^{25}$

\section{Cancer vaccination model}

B16-OVA melanoma cells $\left(2 \times 10^{5}\right.$ cells $)$ were injected subcutaneously into the shaved flank skin of adult female CD45.2 $2^{+}$ mice neonatally infected with $\mathrm{H}$. pylori or $\mathrm{H}$. felis or treated with BHI. Six days later, CD45.1 $1^{+}$OT-1 CD ${ }^{+}$T lymphocytes were isolated from the lymph nodes and spleen using CD8 $\mathrm{a}^{+} \mathrm{T}$ Cell Isolation kit (MACS, Miltenyi Biotec GMBH, Solothurn, Switzerland). OT-1 CD $8^{+}$T cells $\left(1 \times 10^{6}\right)$ were then adoptively transferred into recipient mice by intravenous injection. The following day, $10 \mu \mathrm{g}$ Ova peptide (SIINFEKL) and $50 \mu \mathrm{g}$ CpG provided by Dr Gregory Verdeil (University of Lausanne) in $100 \mu \mathrm{L}$ PBS were injected subcutaneously on the opposite flank to the tumour site in order to immunise the mice. Anti-IFN $\gamma$ neutralising antibodies $(250 \mu \mathrm{g} /$ dose, XMG1.2 clone, BioXcell) were intraperitoneally administrated the day of $\mathrm{OVA}+\mathrm{CpG}$ vaccination and 24 hours later to evaluate the biological role of IFN $\gamma$ in the vaccine-induced tumour control (see online supplemental figures $\mathrm{S} 1 \mathrm{G}$ ). Tumour volumes were measured according to length and width using Vernier callipers from day 7 following tumour engraftment and every 2-3 days thereafter (see online supplemental figure S1C). For flow cytometry analysis, mice were sacrificed 15 days post tumour engraftment.

\section{Antibiotic treatment}

H. pylori infection was eradicated by administering by oral gavage a combination of two antibiotics (amoxicillin $(28.6 \mathrm{mg}$ / $\mathrm{kg} /$ day $)$ and clarithromycin $(14.3 \mathrm{mg} / \mathrm{kg} /$ day $))$ and a proton pump inhibitor (omeprazole $(400 \mu \mathrm{mol} / \mathrm{kg} /$ day)) (see online supplemental figures S1F). ${ }^{46}$ The treatment lasted 7 days. The efficacy of eradication was controlled at sacrifice (see online supplemental figure S2B).

\section{Faecal transplantation}

Adult infected and non-infected mice were treated with enrofloxacin, amoxicillin and clavulanic acid for 3 weeks ${ }^{47}$ and orally administered with $100 \mathrm{mg}$ of faeces from infected mice on a daily basis for 5 days. ${ }^{48}$ Two weeks after the last faeces administration, we started the B16-OVA melanoma model (see online supplemental figure S1E).

\section{CFSE labeling}

OT-1 CD ${ }^{+} \mathrm{T}$ cells pooled from the lymph nodes and spleen of OT-1 mice were washed twice in warm PBS and were then incubated with carboxyfluorescein succinimidyl ester (CFSE, Enzo Life Sciences, Lausen, Switzerland) diluted in warm PBS $(2 \mu \mathrm{M})$ for $10 \mathrm{~min}$ at $37^{\circ} \mathrm{C}$. Cells were then washed once in cold PBS and twice in RPMI supplemented with $10 \% \mathrm{FBS}^{49}$ CFSElabelled cells were used for in vivo OT-1 CD8 ${ }^{+} \mathrm{T}$ cell proliferation experiments and for in vitro cross-presentation assays.

\section{In vivo $0 \mathrm{~T}-1 \mathrm{CD}^{+} \mathrm{T}$ cell proliferation}

Adult CD45.2 $2^{+}$C57BL/6 mice received $2 \times 10^{5}$ B16-OVA cells by subcutaneous injection. Six days later, $1 \times 10^{6}$ CFSE-labelled CD $45.1^{+}$OT-1 $\mathrm{CD}^{+}{ }^{+} \mathrm{T}$ cells were adoptively transferred by intravenous injection. The following day, mice were immunised with $10 \mu \mathrm{g}$ OVA peptide (SIINFEKL) and $50 \mu \mathrm{g}$ CpG in $100 \mu \mathrm{L}$ PBS by subcutaneous injection on the opposite flank to the tumour. Three days postimmunisation, mice were sacrificed, and the tdLNs were collected and processed for flow cytometry analysis. For the analysis of CFSE dilution, cells were stained with antibodies directed towards: CD45.2; CD45.1; CD3 and CD8, see Flow cytometry section in the online supplemental methods for details.

\section{In vivo killing assay}

The in vivo killing assay was performed by the intravenous injection of CFSE-labelled target cells as described by Trompette $e t$ al. ${ }^{50}$ Briefly, splenocytes were loaded with OVA peptide $(200 \mu \mathrm{M})$ or left unloaded and labelled with $0.5 \mu \mathrm{M}$ or $5 \mu \mathrm{M}$ CSFE, respectively. A mixed 1:1 ratio between the two-labelled cell populations was intravenous injected into recipient mice (see online supplemental figure $\mathrm{S} 1 \mathrm{H}$ ). Fourteen hours postinjection, the spleens of recipient mice were recovered and the ratio between the CFSE $^{\text {low }}$ and CFSE ${ }^{\text {high }}$ fraction was determined by flow cytometry to assess killing activity of OT-1 cells.

\section{CD11 $\mathrm{c}^{+} \mathrm{DC}$ isolation}

The spleens of $H$. pylori-infected and non-infected CD45.2 ${ }^{+}$ C57BL/6 adult mice were collected and incubated with Collagenase IV (Sigma) and DNAse (Sigma) for $20 \mathrm{~min}$ at $37^{\circ} \mathrm{C}$. The spleens were then filtered through a $40 \mu \mathrm{m}$ cell strainer and washed in RPMI supplemented with 10\% fetal calf serum (FCS). Cells were washed in MACS buffer and CD11 ${ }^{+}$DCs were isolated using a Pan dendritic cell isolation kit (MACS, Miltenyi Biotec GMBH).

\section{Ex vivo cross-presentation assay}

CFSE-labelled OT- $1 \mathrm{CD} 8^{+} \mathrm{T}$ cells $\left(2 \times 10^{5}\right)$ were cultured in vitro with $2 \times 10^{5} \mathrm{CD}_{11 \mathrm{c}^{+}}$DCs in RPMI supplemented with $10 \%$ FCS, $1 \% \mathrm{P} / \mathrm{S} ; 1 \mathrm{mM}$ sodium pyruvate, $0.05 \mathrm{mM} \beta$-mercaptoethanol and $10 \mathrm{mM}$ HEPES in the presence of different concentrations of OVA protein (Ovalbumin, EndoFit, InvivoGen). After 72 hours of incubation at $37^{\circ} \mathrm{C}$, cells were recovered and analysed by flow cytometry analysis.

\section{Quantification of inflammatory cytokines in the serum}

Using the kit instructions, a broad spectrum of cytokines were measured in the serum of non-infected and infected mice at different time points post $\mathrm{OVA} / \mathrm{CpG}$ immunisation using the LEGENDPlex mouse inflammation panel kit (Biolegend, 
740446). Data were acquired using LSRFortessa cell analyzer (BD, San Jose, California, USA) and analysed using Biolegend's LEGEND's plex data analysis software.

\section{H. pylori IgG ELISA}

H. pylori IgG antibodies were quantified in the plasma of patients, following the protocol for ELISA tests (H. pylori IgG ELISA Kit, Genesis Diagnostics). The optical density (OD) at $450 \mathrm{~nm}$ was read in a microplate reader (Tecan, Infinite M200 Pro).

\section{Statistical analysis}

Student's t-tests (unpaired, two tailed), Mann-Whitney or twoway analysis of variance tests were used to calculate the levels of significance between groups using GraphPad Prism V.8g. Graphs and figure legends are annotated with the level of significance between the tested groups.

\section{Dijon atient cohort}

This cohort contained 60 patients from three French university hospitals with stage IIIB or IV NSCLC who had previously received one or two lines of chemotherapy. All patients were treated with first-line platinum-based chemotherapy. None of the patients had epidermal growth factor receptor, anaplastic lymphoma kinase, B-Raf proto-oncogene serine/threonine kinase or ROS1 oncogenic driven tumours. On progression, they received $3 \mathrm{mg} / \mathrm{kg}$ nivolumab administered intravenously as a single agent every 2 weeks. Tumour response was evaluated by CT scan every four cycles. Tumours were collected, stored and used with informed and written consent from the patients. Clinical characteristics are shown in online supplemental table 1.

\section{Survival curves}

Univariate and multivariate Cox proportional-hazards models were built to estimate HRs with a 95\% CI. Among clinical variables, age, sex, WHO performance status and histology were evaluated and considered not significant ( $p$ value $>0.05$ ).

Survival probabilities were estimated using the Kaplan-Meier method and survival curves were compared using the log-rank test. Statistical analyses were performed using the $\mathrm{R}$ software ((http://www.R-project.org/) and graphs were created using GraphPad Prism V.7.03.

\section{Montreal patient cohort}

Patients with metastatic NSCLC $(n=29)$ undergoing treatment with ICIs were prospectively included (online supplemental table 2). Blood and stool samples from these patients were collected through the CRCHUM lung cancer biobank (Ethics protocol 18.085-17.035, Montreal, Canada). All patients provided informed consent prior to study enrollment. Clinical characteristics are shown in online supplemental table 2.

\section{Acknowledgements We thank Professor Solange Peters (Department of Oncology, CHUV, Lausanne, Switzerland) for her support in developing the proposal supported by the Swiss Cancer Research foundation. We thank Aurelien Trompette for his expertise and guidance with the faecal transplantation and in vivo cytotoxic assays.}

Contributors DV conceived the project and designed the study. PO, LV, ER, FG, BR, GV and DV analysed the data and prepared the manuscript. ER, PO, LV, BM, CB, CT, $C R, M M L, M M, E M$ and EL performed experiments. All authors interpreted the data, discussed the results and revised the manuscript.

Funding This work was supported by the Swiss Cancer Research foundation (KFS4452-02-2018, DV; KFS-4840-08-2019, GV), the Foundation Max Cloëtta (GV), the Foundation Emma Muschamp (DV), the Foundation Porphyrogenis (EM) and by the Faculté de Biologie et Médecine de Lausanne (DV).
Competing interests FG received honoraria for oral communication from Lilly, Sanofi, Amgen, and is an advisory board of Merck Serono, Amgen and Sanofi. The remaining authors declare no conflicts of interest.

Patient consent for publication Not required.

Ethics approval The study protocol was approved by the hospital's ethics committee. The study was performed in accordance with the guidelines of the Declaration of Helsinki.

Provenance and peer review Not commissioned; externally peer reviewed.

Data availability statement Data are available upon reasonable request.

Supplemental material This content has been supplied by the author(s). It has not been vetted by BMJ Publishing Group Limited (BMJ) and may not have been peer-reviewed. Any opinions or recommendations discussed are solely those of the author(s) and are not endorsed by BMJ. BMJ disclaims all liability and responsibility arising from any reliance placed on the content. Where the content includes any translated material, BMJ does not warrant the accuracy and reliability of the translations (including but not limited to local regulations, clinical guidelines, terminology, drug names and drug dosages), and is not responsible for any error and/or omissions arising from translation and adaptation or otherwise.

Open access This is an open access article distributed in accordance with the Creative Commons Attribution Non Commercial (CC BY-NC 4.0) license, which permits others to distribute, remix, adapt, build upon this work non-commercially, and license their derivative works on different terms, provided the original work is properly cited, appropriate credit is given, any changes made indicated, and the use is non-commercial. See: http://creativecommons.org/licenses/by-nc/4.0/.

\section{ORCID iDs}

Francois Ghiringhelli http://orcid.org/0000-0002-5465-8305

Dominique Velin http://orcid.org/0000-0001-6033-5871

\section{REFERENCES}

1 Gopalakrishnan $\mathrm{V}$, Helmink BA, Spencer $\mathrm{CN}$, et al. The influence of the gut microbiome on cancer, immunity, and cancer immunotherapy. Cancer Cell 2018;33:570-80.

2 Vétizou M, Pitt JM, Daillère R, et al. Anticancer immunotherapy by CTLA-4 blockade relies on the gut microbiota. Science 2015;350:1079-84.

3 Gopalakrishnan V, Spencer CN, Nezi L, et al. Gut microbiome modulates response to anti-PD-1 immunotherapy in melanoma patients. Science 2018;359:97-103.

4 Routy B, Le Chatelier E, Derosa L, et al. Gut microbiome influences efficacy of PD-1based immunotherapy against epithelial tumors. Science 2018;359:91-7.

5 Cheng WY, Wu C-Y, Yu J. The role of gut microbiota in cancer treatment: friend or foe? Gut 2020;69:1867-76.

6 Elkrief A, Derosa L, Kroemer G, et al. The negative impact of antibiotics on outcomes in cancer patients treated with immunotherapy: a new independent prognostic factor? Ann Oncol 2019;30:1572-9.

7 Roberti MP, Yonekura S, Duong CPM, et al. Chemotherapy-Induced ileal crypt apoptosis and the ileal microbiome shape immunosurveillance and prognosis of proximal colon cancer. Nat Med 2020;26:919-31.

8 Moyat M, Velin D. Immune responses to Helicobacter pylori infection. World J Gastroenterol 2014:20:5583-93.

9 Altobelli A, Bauer M, Velez K, et al. Helicobacter pylori VacA Targets Myeloid Cells in the Gastric Lamina Propria To Promote Peripherally Induced Regulatory T-Cell Differentiation and Persistent Infection. mBio 2019:10:10:e00261-19.

10 Ricci V, Giannouli M, Romano M, et al. Helicobacter pylori gamma-glutamyl transpeptidase and its pathogenic role. World J Gastroenterol 2014;20:630-8.

11 Arnold IC, Zhang X, Artola-Boran M, et al. BATF3-dependent dendritic cells drive both effector and regulatory T-cell responses in bacterially infected tissues. PLoS Pathog 2019;15:e1007866

12 Engler DB, Reuter S, van Wijck Y, et al. Effective treatment of allergic airway inflammation with Helicobacter pylori immunomodulators requires BATF3-dependent dendritic cells and IL-10. Proc Natl Acad Sci U S A 2014;111:11810-5.

13 Zhuang Y, Cheng P, Liu X-fei, et al. A pro-inflammatory role for Th22 cells in Helicobacter pylori-associated gastritis. Gut 2015;64:1368-78.

14 Chen Y, Blaser MJ. Helicobacter pylori colonization is inversely associated with childhood asthma. J Infect Dis 2008; 198:553-60.

15 Sawalha AH, Schmid WR, Binder SR, et al. Association between systemic lupus erythematosus and Helicobacter pylori seronegativity. J Rheumato/ 2004;31:1546-50.

16 Luther J, Dave M, Higgins PDR, et al. Association between Helicobacter pylori infection and inflammatory bowel disease: a meta-analysis and systematic review of the literature. Inflamm Bowel Dis 2010;16:1077-84.

17 Dellon ES, Peery AF, Shaheen NJ, et al. Inverse association of esophageal eosinophilia with Helicobacter pylori based on analysis of a US pathology database. Gastroenterology 2011;141:1586-92.

18 Oertli M, Noben M, Engler DB, et al. Helicobacter pylori -glutamyl transpeptidase and vacuolating cytotoxin promote gastric persistence and immune tolerance. Proc Natl Acad Sci U S A 2013;110:3047-52 
19 Oertli M, Sundquist M, Hitzler I, et al. DC-derived IL-18 drives Treg differentiation, murine Helicobacter pylori-specific immune tolerance, and asthma protection. J Clin Invest 2012;122:1082-96.

20 Arnold IC, Dehzad N, Reuter S, et al. Helicobacter pylori infection prevents allergic asthma in mouse models through the induction of regulatory T cells. J Clin Invest 2011;121:3088-93.

21 Engler DB, Leonardi I, Hartung ML, et al. Helicobacter pylori-specific protection against inflammatory bowel disease requires the NLRP3 inflammasome and IL-18. Inflamm Bowel Dis 2015;21:854-61.

22 Callahan MK, Postow MA, Wolchok JD. Ctla-4 and PD-1 pathway blockade: combinations in the clinic. Front Oncol 2014:4:385.

23 Murray T, Fuertes Marraco SA, Baumgaertner P, et al. Very late antigen-1 marks functional Tumor-Resident CD8 T cells and correlates with survival of melanoma patients. Front Immunol 2016;7:573.

24 Arnold IC, Zigova Z, Holden M, et al. Comparative whole genome sequence analysis of the carcinogenic bacterial model pathogen Helicobacter felis. Genome Biol Evol 2011;3:302-8.

25 Mager LF, Burkhard R, Pett N, et al. Microbiome-derived inosine modulates response to checkpoint inhibitor immunotherapy. Science 2020;369:1481-9.

26 Kienesberger S, Cox LM, Livanos A, et al. Gastric Helicobacter pylori infection affects local and distant microbial populations and host responses. Cell Rep 2016;14:1395-407.

27 Chen C-C, Liou J-M, Lee Y-C, et al. The interplay between Helicobacter pylori and gastrointestinal microbiota. Gut Microbes 2021;13:1909459-2228.

28 Sivan A, Corrales L, Hubert N, et al. Commensal Bifidobacterium promotes antitumor immunity and facilitates anti-PD-L1 efficacy. Science 2015;350:1084-9.

29 Wilson BE, Routy B, Nagrial A, et al. The effect of antibiotics on clinical outcomes in immune-checkpoint blockade: a systematic review and meta-analysis of observational studies. Cancer Immunol Immunother 2020;69:343-54.

30 lida N, Dzutsev A, Stewart CA, et al. Commensal bacteria control cancer response to therapy by modulating the tumor microenvironment. Science 2013;342:967-70.

31 Uribe-Herranz M, Bittinger K, Rafail S, et al. Gut microbiota modulates adoptive cell therapy via CD8 $\alpha$ dendritic cells and IL-12. JCI Insight 2018;3. doi:10.1172/jci. insight.94952. [Epub ahead of print: 22 Feb 2018].

32 van Wijck Y, de Kleijn S, John-Schuster G, et al. Therapeutic Application of an Extract of Helicobacter pylori Ameliorates the Development of Allergic Airway Disease. J Immunol 2018;200:3027.2-8.

33 Fumet J-D, Richard C, Ledys F, et al. Prognostic and predictive role of CD8 and PD-L1 determination in lung tumor tissue of patients under anti-PD-1 therapy. $\mathrm{Br} J$ Cancer 2018;119:950-60.

34 Oertli M, Noben $M$, Engler DB, et al. Helicobacter pylori $\gamma$-glutamyl transpeptidase and vacuolating cytotoxin promote gastric persistence and immune tolerance. Proc Natl Acad Sci U SA 2013:110:3047-52.
35 Koch KN, Hartung ML, Urban S, et al. Helicobacter urease-induced activation of the TLR2/NLRP3/IL-18 axis protects against asthma. J Clin Invest 2015;125:3297-302.

36 Kalali $B$, Mejías-Luque $R$, Javaheri $A$, et al. $H$. pylori virulence factors: influence on immune system and pathology. Mediators Inflamm 2014;2014:1-9.

37 Kyburz A, Urban S, Altobelli A, et al. Helicobacter pylori and its secreted immunomodulator vacA protect against anaphylaxis in experimental models of food allergy. Clin Exp Allergy 2017;47:1331-41.

38 Al Nabhani Z, Dulauroy S, Marques R, et al. A weaning reaction to microbiota is required for resistance to Immunopathologies in the adult. Immunity 2019;50:1276-88

39 Ivanov II, Atarashi K, Manel N, et al. Induction of intestinal Th17 cells by segmented filamentous bacteria. Cell 2009;139:485-98

40 Chen D, Wu J, Jin D, et al. Fecal microbiota transplantation in cancer management: current status and perspectives. Int J Cancer 2019;145:2021-31.

41 Baruch EN, Youngster I, Ben-Betzalel G, et al. Fecal microbiota transplant promotes response in immunotherapy-refractory melanoma patients. Science 2021;371:602-9.

42 Davar D, Dzutsev AK, McCulloch JA, et al. Fecal microbiota transplant overcomes resistance to anti-PD-1 therapy in melanoma patients. Science 2021;371:595-602.

43 Velin D, Bachmann D, Bouzourene $\mathrm{H}$, et al. Mast cells are critical mediators of vaccine-induced Helicobacter clearance in the mouse model. Gastroenterology 2005:129:142-55.

44 Velin D, Favre L, Bernasconi E, et al. Interleukin-17 is a critical mediator of vaccineinduced reduction of Helicobacter infection in the mouse model. Gastroenterology 2009;136:2237-46.

45 Begka C, Pattaroni C, Mooser C, et al. Toll-Interacting protein regulates immune cell infiltration and promotes colitis-associated cancer. iScience 2020;23:100891.

46 van Zanten SJOV, Kolesnikow T, Leung V, et al. Gastric transitional zones, areas where Helicobacter treatment fails: results of a treatment trial using the Sydney strain mouse model. Antimicrob Agents Chemother 2003;47:2249-55.

47 Yadava K, Pattaroni C, Sichelstiel AK, et al. Microbiota promotes chronic pulmonary inflammation by enhancing IL-17A and autoantibodies. Am J Respir Crit Care Med 2016;193:975-87.

48 D'Amato A, Di Cesare Mannelli L, Lucarini E, et al. Faecal microbiota transplant from aged donor mice affects spatial learning and memory via modulating hippocampal synaptic plasticity- and neurotransmission-related proteins in young recipients. Microbiome 2020;8:140.

49 Moyat M, Bouzourene $\mathrm{H}$, Ouyang W, et al. IL-22-induced antimicrobial peptides are key determinants of mucosal vaccine-induced protection against $\mathrm{H}$. pylori in mice. Mucosal Immunol 2017;10:271-81.

50 Trompette A, Gollwitzer ES, Pattaroni C, et al. Dietary Fiber Confers Protection against Flu by Shaping Ly6c Patrolling Monocyte Hematopoiesis and CD8 ${ }^{+} \mathrm{T}$ Cell Metabolism. Immunity 2018:48:992-1005. 